Cite this: RSC Adv., 2014, 4, 10303

Received 23rd December 2013 Accepted 31st January 2014

DOI: $10.1039 / c 3 r a 47915 c$

www.rsc.org/advances

\section{Sonochemically-induced spectral shift as a probe of green fluorescent protein release from nano capsules}

\author{
Ulyana Shimanovich, ${ }^{\text {a }}$ Anna Munder, ${ }^{\mathrm{b}}$ Nuno G. Azoia, ${ }^{\mathrm{c}}$ Artur Cavaco-Paulo, ${ }^{\mathrm{c}}$ \\ Arie Gruzman, ${ }^{\star b}$ Tuomas P. J. Knowles ${ }^{a}$ and Aharon Gedanken ${ }^{\star b}$
}

Encapsulation in the form of micro and nanocapsules is an attractive route for controlling the delivery and release of active proteins and peptides. Many approaches exist to probe the morphology of such capsules as well as their mechanisms of formation. By contrast, the release of proteins from such components in a complex biological environment has been challenging to probe directly. In this paper we show that the spectral differences between green fluorescent protein (GFP) in capsules and in its free form can be used to monitor in situ the release of the protein from the confinement of capsules. These findings represent a new route towards engineering the spectral characteristics of GFP through physical rather than chemical means. We demonstrate the use of GFP protein capsules for monitoring in real time the release of protein in live cells by exposing rat $L 6$ myotubes to protein capsules. The GFP spheres with a blue fluorescent signal dissociate inside the L6 myotubes to individual GFP molecules with a change in fluorescent signal from blue to green. These sensitive spectral characteristics enabled us to resolve the dissociation of capsules inside the cells in both time and space. We discuss the implications of our results for quantifying parameters crucial for the delivery of proteins in biological environments.

\section{Introduction}

The green fluorescent protein (GFP) was discovered in 1960's by Shimomura et $a l^{\mathbf{1}}$ as a companion protein molecule to the aequorin protein isolated from Aequorea jellyfish. The green fluorescent protein of the jellyfish Aequorea victoria is an unusual protein with strong visible absorbance and fluorescence from a p-hydroxybenzylidene-imidazolidinone chromophore $^{2}$, which is generated by cyclization and oxidation of the protein's own Ser-Tyr-Gly sequence at positions 65-67, where the overlap of three amino acids generates $\pi-\pi^{*}$ interactions

${ }^{a}$ University of Cambridge, Department of Chemistry, Lensfield road, Cambridge, CB2 1EW,UK.E-mail: us249@cam.ac.uk

${ }^{b}$ Bar-Ilan University, Department of Chemistry, Ramat-Gan, 52900, Israel. E-mail: gedanken@mail.biu.ac.il; gruzmaa@biu.ac.il

'University of Minho, IBB-Institute for Biotechnology and Bioengineering, Centre of Biological Engineering, Campus de Gualtar, 4710-057, Braga, Portugal leading to fluorescence at visible wavelengths. The GFP's excitation band is in the blue, at $395 \mathrm{~nm}$ (major peak) and $475 \mathrm{~nm}$ (minor peak), and the emission is in the green region of fluorescent spectra with a peak at $509 \mathrm{~nm}$. Since the discovery of the GFP protein in 1960's, the popularity of GFP has grown as a gene reporter $^{3,4}$ in the fields of molecular biology and biotechnology as well as an imaging agent in the fields of protein chemistry and cellular biology ${ }^{6}$. A major extension to the capabilities of GFP has arisen through the tailoring of it photophysical properties to generate emission in ranges other than green, in particular yellow and orange. Until now, however, such spectral engineering has explored, in majority of the cases, mutations in core sequence of GFP chromophore ${ }^{7,8}$. GFP has been used to monitor the release of protein in the cytosol of living cells from biodegradable polyelectrolyte capsules ${ }^{9,10}$ fabricated to encapsulate GFP inside such structures as cargo. This type of approach is based on the release of the fluorescent protein from the core of the polymeric capsules as result of either laser irradiation (from light-responsive electrolyte capsules) or by enzymatic degradation (from biodegradable capsules). We present an alternative strategy to introduce GFP into living cells and to monitor its release, namely to use the GFP protein itself as a natural building block for capsule construction as well as to tailor the fluorescent properties of the capsules. In this paper, we show a strong but reversible shift in the GFP emission spectrum emerges as a result of protein confinement and reversible cross-linking upon protein capsule formation.

\section{Materials and Methods}

\section{Sonochemical synthesis of GFP capsules}

For the preparation of GFP proteinaceous nanospheres, the following reactants were used: 1) A $1 \% \mathrm{w} / \mathrm{v}$ aqueous solution of GFP (recombinant green fluorescent protein; molecular weight $27 \mathrm{kDa}$; Alpha-Diagnostic); 2) Dodecane (98.0\% Fluka). The volume ratio of the $1 \%(\mathrm{w} / \mathrm{v})$ GFP aqueous solution and dodecane was kept constant, 3:2, respectively. In brief, dodecane $(6.7 \mathrm{~mL}, 98.0 \%$ Fluka) was layered over a water solution 
$(10 \mathrm{~mL})$ of $1 \% \mathrm{w} / \mathrm{v}$ protein. The GFP spheres were synthesised with a high-intensity ultrasonic probe (Sonic and Materials, VC$600,20 \mathrm{kHz}, 0.5 \mathrm{in}$. Ti horn, at 78\% amplitude). The volume of the acoustic chamber was $25 \mathrm{~mL}$ and the total volume of all the ingredients was $16.7 \mathrm{~mL}$. The bottom of the high-intensity ultrasonic horn was positioned at the aqueous-organic interface, employing an acoustic power of about $150 \mathrm{~W} \mathrm{~cm}^{-3}$ with an initial temperature of $22{ }^{\circ} \mathrm{C}$ in the reaction cell. The sonication lasted for $3 \mathrm{~min}$ at $22{ }^{\circ} \mathrm{C}$ by using an ice-cooling bath to maintain the low temperature. At the end of the reaction, the temperature in the reaction cell reached $28{ }^{\circ} \mathrm{C}$. The rise in temperature from 22 to $28^{\circ} \mathrm{C}$ (measured by a thermocouple) did not lead to the proteins degradation. The temperature in the reaction cell should not rise above the denaturation temperature of the proteins $\left(58{ }^{\circ} \mathrm{C}\right)$. A separation flask was used to separate the product from the mother solution. The separation was accomplished within a few minutes due to the lower density of the proteinaceous capsules, relative to the density of water. To obtain a more complete separation of the proteinaceous spheres from the mother solution, the separation flasks were placed in a refrigerator $\left(4{ }^{\circ} \mathrm{C}\right)$ for $24 \mathrm{~h}$. After the separation, the residual aqueous phase and the organic solvent (dodecane/ canola/soyabean oil) were removed and the product was resuspended in water.

\section{GFP proteinaceous capsules efficiency studies}

For all studies (morphological, size, efficiency studies) the following concentrations of native GFP protein as well as GFP nanospheres were used: GFP protein with concentration $100 \mu \mathrm{g} / \mathrm{ml}$ and GFP nanospheres concentration of $87 \mu \mathrm{g} / \mathrm{ml}$. The efficiency of the GFP capsules formation for the sonochemical and microfluidic methods in converting a native GFP protein into GFP proteinaceous spheres was measured using UV spectroscopy. The protein absorption peak was detected at $280 \mathrm{~nm}$. To calculate the efficiency, the amount of the free protein was subtracted from the total amount of protein introduced in reaction cell. The measurements show that about $89 \%$ of the initial amount of the protein was converted into proteinaceous spheres following ultrasound treatment and around $87 \%$ due to microfluidic droplet creation.

\section{Circular dichroism}

The conformational changes in the GFP protein due to ultrasonic treatment was studied using the Circular Dichroism technique (Chirascan, AppliedBiophysics). The two CD spectra were compared: the spectra of native GFP and the spectra of GFP spheres. The data obtained from the CD measurements were analyzed using the K2d program and the percentage of the protein secondary structure was calculated. Data values from CD spectra for calculation in $\operatorname{deg} \mathrm{cm}^{2} \mathrm{dmol}^{-1}$ multiplied by 0.001. CD spectrum data points were taken into account, ranging from $200 \mathrm{~nm}$ to $240 \mathrm{~nm}$. The calculation shows a change in the percentage of an $\alpha$-helix component of the protein upon spheridization.

\section{Molecular Dynamic Simulations}

The molecular dynamic simulations provide the information about the main conformation changes of GFP protein due to ultrasonic treatment. The 3D simulations of the structural changes of GFP protein caused by water, oil and water-oil solutions were performed with the GROMACS 4.0.7 ${ }^{21}$ ) package using the GROMOS 53A6 force field. For the simulations with water/oil mixtures, the systems were solvated using a preequilibrated box of dodecane. The mixture of protein and dodecane, or the protein alone for the studies in water, was solvated using a pre-equilibrated box of water. The system size was chosen according to the minimum image convention, taking into account a cut-off of $1.4 \mathrm{~nm}$. The bond lengths of the proteins were constrained with lincs and those for water with settle. Non-bonded interactions were calculated using a twinrange method, with short and long range cut-offs of 0.8 and $1.4 \mathrm{~nm}$, respectively. Neighbor searching was carried out up to $1.4 \mathrm{~nm}$ and updated every five steps. A time step of integration of $2 \mathrm{fs}$ was used. A reaction field correction for the electrostatic interactions was applied using a dielectric constant of 54. The single point charge (SPC) model ${ }^{22}$ was used for water molecules. The initial systems were energy minimized for 2000 steps using the steepest descent method, with all the heavy atoms harmonically restrained using a force constant of $103 \mathrm{~kJ} / \mathrm{mol}$ $\mathrm{nm}^{2}$. The systems were initialized in the canonical ensemble (NVT) for $50 \mathrm{~ns}$, with all heavy atoms harmonically restrained using a force constant of $103 \mathrm{~kJ} / \mathrm{molb} \mathrm{nm}^{2}$. The simulation was then continued for $50 \mathrm{~ns}$ in the isothermal-isobaric ensemble (NPT), with the heavy atoms harmonically restrained with the same force constant. Finally, for enabling the equilibration of the system's properties, the simulations were further extended in the NPT ensemble with positional restrains applied to the C atoms. Pressure control was implemented using the Berendsen barostat, with a reference pressure of 1 bar, 0.5 ps of relaxation time and isothermal compressibility of $4.510^{5}$ bar. Temperature control was set using the $\mathrm{V}$ rescale thermostat at $300 \mathrm{~K}$. The protein and the solvent molecules were coupled in separated heat baths, with temperature coupling constants of $0.025 \mathrm{ps}$ in the first two initialization steps and with $0.1 \mathrm{ps}$ for the rest of the simulations. Each simulation was carried out for $40 \mathrm{~ns}$.

\section{Fluorescent properties of native GFP and GFP capsules}

The excitation and emission spectra of the nano and micron sized GFP spheres were measured and compared to the spectra of the native protein. Under physiological conditions (e.g., $\mathrm{T}=25$ ${ }^{\circ} \mathrm{C}, \mathrm{pH}=7$ ) the chromophore has a neutral form and its major excitation at $396 \mathrm{~nm}$ giving rise to the green fluorescence with a measured maximum at $509 \mathrm{~nm}$. Quantum yield $(\mathrm{QY})=0.674$, Molar extinction coefficient $(\varepsilon)=23162(9121) \mathrm{M} \mathrm{cm}^{-1}$. Excitation and emission spectra of GFP spheres formed by microfluidic method (excitation was detected at 395 and emission at 508). $\mathrm{QY}=0.643, \varepsilon=23124(9137) \mathrm{M} \mathrm{cm}^{-1}$. Excitation and emission spectra of GFP spheres formed sonochemically (excitation maxima are measured at $353 \mathrm{~nm}$ ). Emission of the GFP spheres was conducted with a maximum at $414 \mathrm{~nm}$. QY=0.68, $\varepsilon=18867 \mathrm{M} \mathrm{cm}^{-1}$. 


\section{GFP proteinaceous capsules stability}

Stability of GFP spheres was studied in aqueous Carboxypeptidase A solution. The GFP capsules' solution was added into the Tris buffer solution (25 mM Tris HCl Buffer with $500 \mathrm{mM}$ Sodium Chloride, $\mathrm{pH}=7.5$ at $25{ }^{\circ} \mathrm{C}$ ) of Carboxypeptidase A-II enzyme (Carboxypeptidase-A from bovine from bovine pancrease Type II (CPA-II), Sigma-Aldrich). The initial concentration of GFP protein in GFP spheres was calculated to be $87 \mu \mathrm{g} /$ $\mathrm{ml}$. The concentration of Carboxypeptidase A used for stability test was 0.2 units per $1 \mathrm{ml}$ protein spheres solution.

\section{Introduction of GFP nanospheres into L6 muscle cells}

Two separate sets of experiments were performed: 1) L6 myotubes were incubated with pristine GFP protein for 1, 3.5 and 24 hours. The main goal of this experiment was to check if GFP protein in its native form is able to penetrate the L6 myotubes body and emit green fluorescent signal. 2) L6 myotubes were incubated with GFP nanospheres for 1, 3.5 and 24 hours. A sub clone of rat skeletal muscle L6 cells (Curtsey of Prof. Shlomo Sasson, the Hebrew University of Jerusalem, Jerusalem, Israel), selected for high fusion potential was used. Cells were grown in a $\alpha$-MEM supplemented with $1 \mathrm{~g} / \mathrm{L}$ D-glucose, $10 \%$ (V/V) FCS, $100 \mathrm{u} / \mathrm{ml}$ penicillin $\mathrm{G}$ and $100 \mathrm{mg} / \mathrm{ml}$ streptomycin, in a 95\%:5\% air/CO2 humidified incubator at $37^{\circ} \mathrm{C}$. Differentiation of myocytes into myotubes was induced with $2 \%$ (V/V) FCS, as previously described ${ }^{23}$. The culture medium was then changed at least every $48 \mathrm{~h}$. L6 cells were incubated with GFP nanospheres for $1,3.5$ and 24 hours at $37{ }^{\circ} \mathrm{C}$. After the incubation, the cells were washed 3 times with a PBS $1 \mathrm{x}$ buffer solution in order to remove the residual GFP spheres. The cell culture was cross-linked on a glass-slide with $4 \%$ para-formaldehyde and then microscopically analyzed.

\section{Confocal, light and SEM microscopy analysis}

For light and confocal microscopy (Confocal, Zeiss Microscope), samples were prepared by depositing the aqueous dispersions, without further purification, onto a glass slide. The GFP spheres were analyzed and characterized by SEM (scanning electron microscopy, JSM-840, JEOL), fluorescent and light microscopy (Apo-Tome microscope, Zeiss), confocal microscopy (Confocal, Zeiss Microscope). For confocal microscopy analysis of the spheres, the following lasers were used: GFP (green fluorescent signal; Argon laser 458/477/488/514 nm, blue signal; UV $405 \mathrm{~nm}$ ) and DIC. The $z$-stack confocal microscopy images were reconstructed using "Imaris" (Bitplane) image analysis software and 3D images of GFP protein capsules were obtained.

\section{Results and Discussions}

To explore the potential of nano confinement to engineer the spectral characteristics of GFP, we formed GFP capsules both by ultrasonic emulsification and microfluidic emulsification. In ultrasonic emulsification, an aqueous protein solution is brought into contact with an immiscible oil phase. Subsequent application of ultrasonic energy leads to the formation of oil in water droplets where the amphiphilic proteins act as surfactants and localize at the interface, and are able to crosslink through hydrogen bonding to form a stable capsule ${ }^{11-16}$. In microfluidic emulsification, by contrast, droplets are created through multi-phase flow in a droplet maker junction within a microfluidic chip without any application of external cavitation $^{17-20}$.

The fraction of GFP converted to capsules was measured by monitoring the concentration of free protein by UV-absorption, revealing that in both cases an encapsulation efficiency of over $85 \%$. The schematic representation of the sonochemical GFP capsules formation is shown in Fig. 1a. The resulting sonochemically and microfluidically formed GFP protein capsules filled with organic solvent in its inner part. However, the initial dissociation of protein capsules formed by the microfluidic method was detected immediately after the synthesis was accomplished, with complete dissociation taking place over three days. Sonochemically synthesized capsules were stable for significantly longer, with complete disintegration taking place over a timescale of several months. The increased stability of the sonochemical capsules is consistent with the idea that this process introduces significant structural changes to the proteins and gives rise to strong protein-protein interactions $\mathbf{s}^{\mathbf{1 1}}$.

Furthermore, we studied the impact of different organic solvents on the encapsulation efficiency as well as on the size of the sonochemically formed GFP capsules (See scheme for mechanism of sonochemical GFP sphere formation in Fig. 2a). A striking difference in average size and efficiency of capsule formation was observed for dodecane, canola and soybean oils. The effect of organic solvents, with different viscosity, on GFP capsules is shown in Fig. 1b. An increase in viscosity of the oils leads to reduction in the efficiency of protein capsule formation, while an inverse trend is observed for average size. The average size of GFP spheres was observed to increase from $235 \mathrm{~nm}$ to $322 \mathrm{~nm}$ with increase in viscosity of organic solvent. The observed results are in good agreement with average size of GFP spheres (about $240 \mathrm{~nm}$ ) that was reported elsewhere ${ }^{\mathbf{1 1}}$. This observation correlates with the stability of the capsules: the time to dissociate was found to be significantly dependent on the nature of the oil phase (3.5 days for canola oil vs. few months for dodecane-filled capsules) for more viscous solutions, suggesting that protein capsule dissociation is strongly affected by the oil-water interface. Interestingly, it is well documented that the formation of cavitation bubbles is strongly dependent on the viscosity of the solvent ${ }^{20}$. The correlation between the yield of the protein capsules and the organic solvent viscosity can be seen as a consequence of this trend.

Both emulsification methods can be used to cover a range of sizes of the formed capsules. GFP spheres formed by microfluidics are approximately $8 \mu \mathrm{m}$ size and the average size of the sonochemically formed GFP spheres is approximately $300 \mathrm{~nm}$. The SEM micrograph of nano-sized GFP capsules and light microscopy image of micron-sized of microfluidically formed capsules are shown in Fig. $2 \mathrm{c}$ and $\mathrm{d}$, respectively. While the average size of sonochemically formed sphere is regulated by the mean of intensity of ultrasonic waves and viscosity of organic solvent, the size of microfluidically synthesized capsules is determined by the width of microfluidic nozzle. 


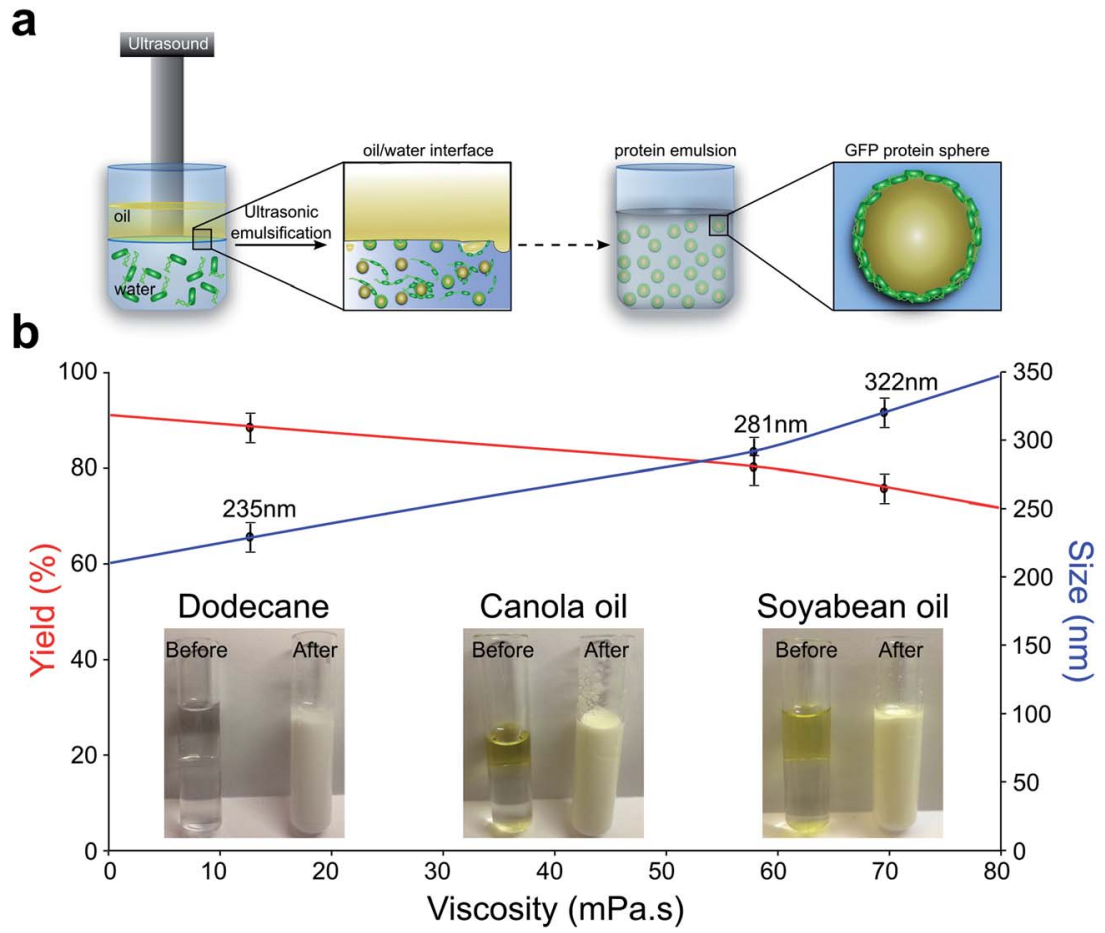

Fig. 1 (a) Schematic representation of sonochemical formation of GFP proteinaceous capsule; (b) digraph showing the change in yield and average size of sonochemically formed GFP capsules as a function of organic solvent viscosity. Images of precursor solution containing dodecane, canola and soybean oils before and after GFP capsules creation is shown at the bottom of the graph.

Examination of the spectral characteristics of the GFP protein in these two different structures revealed striking differences. While the spectra acquired from the microfluidic capsules were indistinguishable from those obtained from the native protein, a marked change was observed for the sonochemically formed capsules. Indeed, the emission was found to be blue-shifted by more than $100 \mathrm{~nm}$.

We further studied dependence of morphology of the sonochemically and microfluidically formed GFP protein capsules by light, scanning electron and confocal microscopy (Fig. 2a, b, c, and $\mathrm{d}$ ). The three dimensional reconstruction of $z$-stack confocal microscopy images unveil the localization of GFP protein in the shell of protein capsule's structure. The confocal microscopy analysis was probed on sonochemically formed GFP protein capsules, shown in Fig. 2a, using 350/450 excitation/ emission wavelengths and microfluidically formed GFP protein capsules, shown in Fig. 2b, were analyzed using 470/570 nm wave lengths (See Methods section).

To investigate further the structural changes in the GFP protein as a consequence of ultrasonic spheridization, the changes in the secondary structure of native GFP protein and GFP in protein capsules were monitored using circular dichroism (CD) measurements (See Methods Section). The results are presented in Fig. 2e. Interestingly, the $\alpha$-helix content before the sonochemical spheridization was found to be around $12 \%$ and after this treatment reduced to $4 \%$. Furthermore, the $8 \%$ increase in random coil confirmation was detected for spherical form of GFP protein, implying that $8 \%$ of the $\alpha$-helix content was transformed into random coil (disordered) state. In order to probe whether the conformational changes of GFP protein due to ultrasonic emulsification or affected by presence of organic solvent, we investigated the role of the oil-water interface on the molecular level structure of GFP by means of molecular dynamic simulations (MD) ${ }^{21,22}$. The MD results are presented in Fig. 2e. The simulations show that there is a small change between the protein structure in a water solution and in a water/dodecane solution. The results of MD simulations further show that the $\alpha$-helix content of the protein is not primarily reduced due to the change in the hydrophobicity of the environment. The $\alpha$-helix GFP component changed its position slightly inside the $\beta$-barrel core. This small change is not sufficient to lead to the blue shift in the GFP fluorescent spectra (See Fig. 2f). In order to obtain a change in the fluorescence of a GFP, the surrounding environment of chromophore has to be changed and indeed our results show that ultrasonic treatment is necessary to convert the green signal of the GFP into blue. Under elevated temperature $\left(40{ }^{\circ} \mathrm{C}\right)$ and mechanical shear, created by passing ultrasonic waves through the biphasic mixture, the GFP protein can partially unfold, exposing its chromophore to the solvent, with a distinct reduction in the fluorescence intensity of the chromophore accompanied by a blue shift.

In order to obtain a more detailed understanding of whether the temperature raise, ultrasonic waves or combination of all of the extrinsic factors, increase in temperature, ultrasonic waves and presence of organic solvent, lead to changes in secondary structure and fluorescent properties of GFP, we investigated the effect of each parameter on GFP protein separately. Increase in 

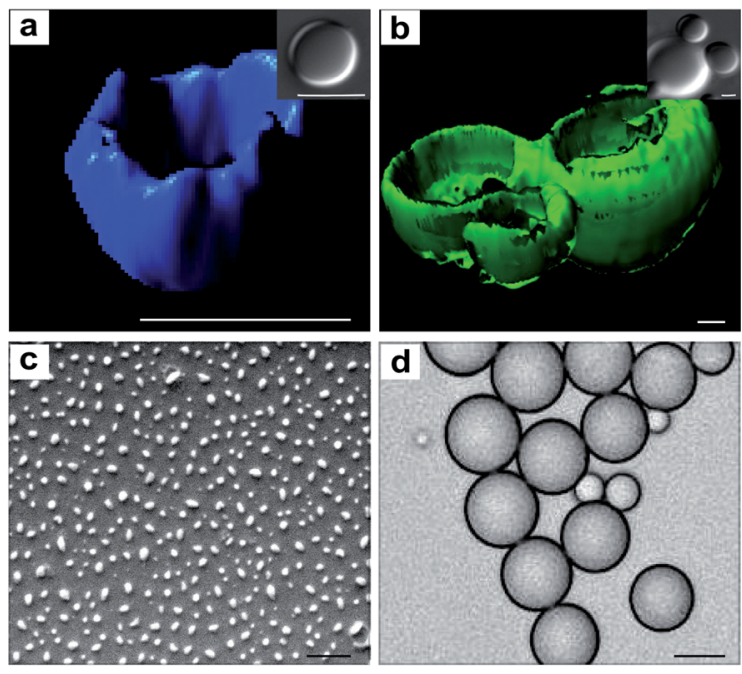

e

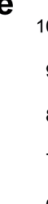

(\%)

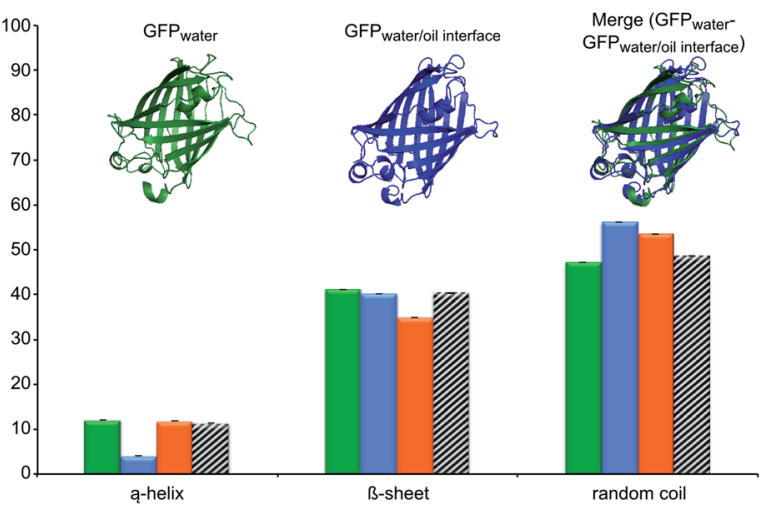

GFP protein conformation

\section{$\mathbf{f}$}

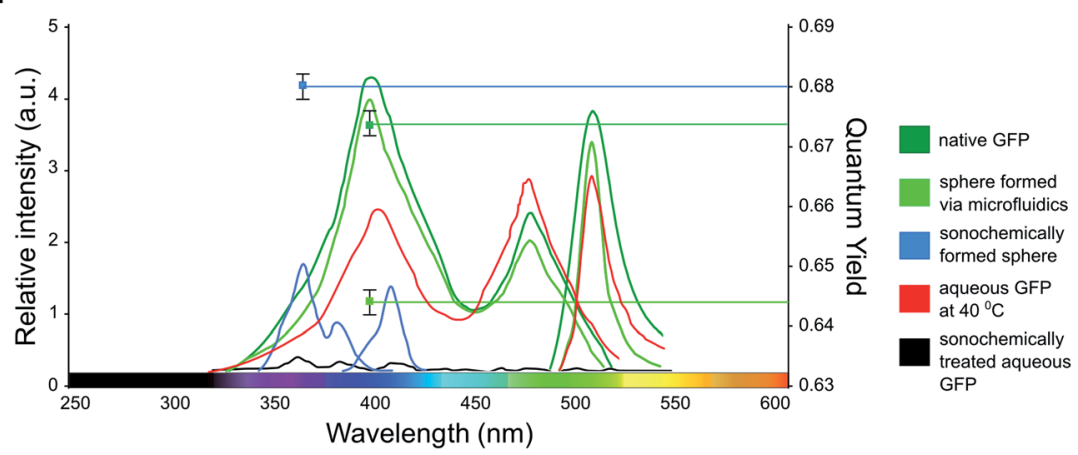

Fig. 2 Confocal images of reconstructed z-stack 3D structures of sonochemically (a) and microfluidically (b) formed GFP spheres. The images of sonochemically prepared GFP blue sphere obtained using fluorescent filter with $350 / 450 \mathrm{~nm}$. excitation/emission wavelengths and for microfluidically formed spheres filters with $470 / 570 \mathrm{~nm}$. excitation/emission were used. Scale Bars $=1 \mu \mathrm{m}$. (c) Scanning electron microscopy (SEM) image of GFP nanospheres formed via sonochemical method. Scale Bar $=1 \mu \mathrm{m}$ (d) Light microscopy image of GFP microsphere formed via microfluidic device. Scale Bar $=10 \mu \mathrm{m}$. (e) Schematic diagram of GFP protein secondary structure before and after spheridization. On the top of the diagram the MD simulation results of GFP in water and water/oil interface are presented. (f) Excitation and emission spectra of native GFP protein, sonochemically formed GFP capsules and microfluidically formed capsules.

the temperature up to $40{ }^{\circ} \mathrm{C}$ without the presence of the organic solvent led to a decrease in the intensity at $395 \mathrm{~nm}$ and increase at $470 \mathrm{~nm}$ in the GFP excitation spectrum with a of overall fluorescence intensity (Fig. 2e). The changes in the fluorescent spectra were accompanied by changes in protein secondary structure, in particular a $15 \%$ decrease in $\beta$-sheet and $1.7 \%$ in $\alpha$-helix contents (Fig. 2f). After the aqueous GFP solution was exposed to ultrasonc waves, a decrease in the fluorescence intensity was observed, accompanied by a decrease of $2 \%$ and $6 \%$ in the $\beta$-sheet and the $\alpha$-helix contents, respectively, but without a blue shift in the fluorescence spectrum. This finding indicates that blue-shifted fluorescence spectral changes of GFP protein originated only from combination of sonochemical treatment with presence of organic solvent and increase in temperature. This combination is likely to promote the partial unfolding of the protein and favour non-covalent 
inter-molecular contacts that can stabilise the capsule and influence the structure of the fluorphore and hence the optical properties of the protein in the nanocapsular form.

The stability of the sonochemically formed GFP nanospheres and its fluorescent signal was probed in the presence of degrading enzyme, Carboxypeptidase-A (See Methods). Carboxypeptidase A (CPA-II) is a digestive enzyme that hydrolyses the carboxy-terminal peptide bond in protein polypeptide chain. The effect of presence CPA-II in solution on GFP spheres stability was studied by UV spectroscopy following the tryptophan absorption maxima at $294 \mathrm{~nm}$. The tryptophan content was measured in the lower residue phase of the aqueous GFP spheres solution. The increase in tryptophan content concentration in lower aqueous phase indicates that GFP protein molecules were hydrolyzed and GFP nano-sized capsules were destroyed. After 18 hours of incubation GFP spheres in CPA-II solution, $50 \%$ of the spheres were destroyed. When the GFP spheres were incubated with enzyme for more than 36 hours $98 \%$ of the spheres were destroyed (See Fig. 3a). The sonochemically formed GFP spheres were found to be stable in the presence of enzymes for less than 40 hours. In order to study the behavior of GFP native protein as well as GFP spheres inside the cells, both native GFP and GFP proteinaceous capsules were introduced into the L6 myotubes (See Methods section).

We propose now to exploit unusual spectral properties of the sonochemically formed proteinaceous GFP capsules as a carrier with environmentally sensitive fluorescence reporting to explore the rates and spatial sub-cellular localisation of protein release subsequent to deliver in nanocapsules. To this effect, we probed uptake of GFP nanocapsules into rat immortalized L6 myotube cells $^{\mathbf{2 3 , 2 4}}$ and monitored the release of free GFP through the accompanying spectral shift. Due to the high signal to noise ratio afforded by tracking species with different emission spectra, we are able to localize both encapsulated and released proteins using confocal microscopy to a sub-cellular resolution in live cells.

A control experiment shows that no fluorescent signal was detected when the native molecules of GFP protein were exposed to cells (See Experimental Section) due of the low efficiency of the internalization of GFP in the monomeric form and as a consequence of the dilution of any GFP inside the cell to the entire cytoplasmic volume. However, when the sonochemically formed GFP nano-sized capsules were introduced into the cells, after $1 \mathrm{~h}$ incubation of only blue signal, emitted from the GFP nanospheres, was detected as shown in Fig. $3 \mathrm{~b}$. When the incubation time was increased from 1 hour to 3.5 hours a fraction of the internalized GFP capsules was found to disintegrate and both blue and green fluorescent signals were obtained. The blue signal originates from to GFP nano sized capsules and the green signal from the released native GFP molecules. After 24 hours of incubation time the blue signal emitted from GFP spheres disappeared and only the green signal related to native GFP protein was detected inside the cells. The increase in incubation time to 48 hours resulted in the disappearance of both signals, blue and green. This is interpreted as due to the hydrolysis of the GFP protein.
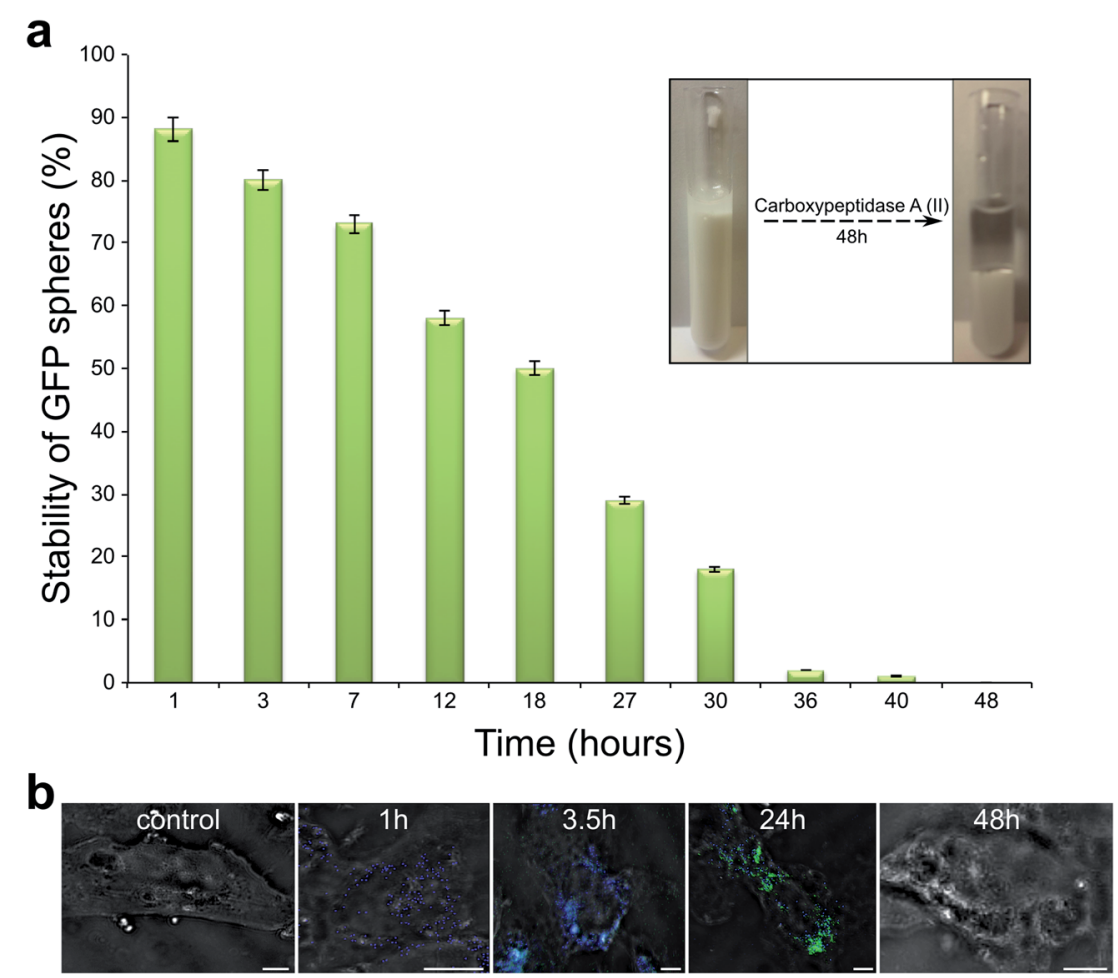

Fig. 3 (a) Stability studies for sonochemically formed GFP nano sized capsules. Stability was checked in presence of CPA-II enzyme as a function of time. On the right corner the images of GFP capsules solutions before and after enzymatic treatment are shown. (b) Confocal microscopy images of L6 cells and L6 cells incubated with sonochemically formed GFP capsules for 1, 3.5, 24 and 48 hours. Scale Bars $=5 \mu$. 


\section{Conclusions}

In conclusion, the present study demonstrates the conversion of the characteristic green fluorescent signal of a GFP protein into blue emission using high intensity ultrasonic waves. This physical engineering of the emission spectra of fluorescent proteins opens up a new avenue for tailoring photophysical properties of proteins in addition to the conventional chemical methods. The reversible spectral shift furthermore can form the basis for a probe of the uptake and release of proteins within cells. The change from blue to green fluorescent signal, due to dissociation of GFP nanospheres inside the cells, was detected within 24 hours. Understanding and controlling the behavior of GFP fluorescent signal opens the variety of possible applications in the field of nanotechnology and cellular biology. A particularly exciting prospect is the possibility for GFP nanosized capsules to serve simultaneously as a delivery agent and an imaging agent.

\section{Competing Interests}

The authors declare that they have no competing financial interests.

\section{References}

1 O. Shimomura, Discovery of green fluorescent protein (gfp) (nobel lecture), Angewandte Chemie International Edition., 2009, 48, 5590-5602.

2 J. J. Thor, T. Gensch, K. J. Hellingwerf and L. N. Johnson, Phototransformation of green fluorescent protein with uv and visible light leads to decarboxylation of glutamate 222, Nature Structural Biology, 2002, 9, 37-41.

3 R. E. Zordan, Y. Ren, S.-J. Pan, G. Rotondo, A. De Las Penas, J. Iluore and B. P. Cormack, Expression plasmids for use in candida glabrata, G3: Genes-Genomes-Genetics, 2013, 3, 1675-1686.

4 R. Niedz, M. Sussman and J. Satterlee, Green fluorescent protein: an in vivo reporter of plant gene expression, Plant Cell Reports., 1995, 14, 403-406.

5 R. Weissleder and V. Ntziachristos, Shedding light onto live molecular targets, Nature Medicine, 2003, 9, 123-128.

6 G. H. Patterson and J. Lippincott-Schwartz, A photoactivatable gfp for selective photolabeling of proteins and cells., Science., 2002, 297, 1873-1877.

7 B. L. Grigorenko, A. V. Nemukhin, I. V. Polyakov and A. I. Krylov, Triple-decker motif for red-shifted fluorescent protein mutants, The Journal of Physical Chemistry Letters., 2013, 4, 1743-1747.

8 A. Pakhomov, S. Tretyakova and V. Martynov, The influence of chromophore-protein interactions on spectroscopic properties of the yellow fluorescent protein, Doklady Biochemistry and Biophysics., 2012, 445, 207-209.

9 S. Carregal-Romero, M. Ochs, P. Rivera-Gil, C. Ganas, A. Pavlov, G. B. Sukhorukov and W. J. Parak, NIR-light triggered delivery of macromolecules into cytosol, Journal of Controlled Release., 2012, 159, 120-127.
10 P. Rivera-Gil, S. De Koker, B. G. De Geest and W. J. Parak, Intracellular processing of proteins mediated by biodegradable polyelectrolyte capsules, Nano Letters., 2009, 9, 4398-4402.

11 U. Angel (Shimanovich), D. Matas, S. Michaeli, A. CavacoPaulo and A. Gedanken, Microspheres of mixed proteins, Chem. Eur. J., 2010, 16, 2108-2114.

12 U. Shimanovich, V. Volcov, D. Eliaz, A. Aizer, S. Michaeli and A. Gedanken, Stabilizing rna by the sonochemical formation of rna nanospheres, Small., 2011, 7, 1068-1074.

13 U. Shimanovich, D. Eliaz, A. Aizer, I. Vayman, S. Michaeli, Y. Shav-Tal and A. Gedanken, Sonochemical synthesis of DNA nanospheres, ChemBioChem., 2011, 12, 1678-1681.

14 M. W. Grinstaff and K. S. Suslick, Air-filled proteinaceous microbubbles: Synthesis of an echo-contrast agent, PNAS., 1991, 88, 7708-7710.

15 K. S. Suslick, M. Grinstaff, K. Kolbeck and M. Wong, Characterization of sonochemically prepared proteinaceous microspheres, Ultrason. Sonochem., 1994, 1, S65-S68.

16 U. Shimanovich, I. D. Tkacz, D. Eliaz, A. Cavaco-Paulo, S. Michaeli and A. Gedanken, Encapsulation of rna molecules in bsa microspheres and internalization into trypanosoma brucei parasites and human U2OS cancer cells, Adv. Func.Mat., 2011, 21, 3659-3666.

17 (a) J. J. Agresti, E. Antipov, A. R. Abate, K. Ahn, A. C. Rowat, J.-C. Baret, M. Marquez, A. M. Klibanov, A. D. Griffiths and D. A. Weitz, Ultrahigh-throughput screening in drop-based microfluidics for directed evolution, PNAS., 2010, 107, 4004-4009; (b) G. M. Whitesides, The origins and the future of microfluidics, Nature., 2006, 442, 368-373.

18 H. A. Stone, Microfluidics: Tuned-in flow control, Nat Phys., 2009, 5, 178-179.

19 M. Prevot, A. L. Cordeiro, G. B. Sukhorukov, Y. Lvov, R. S. Besser and H. Moehwald, Microfluidic system incorporating layer-by-layer nanofabricated capsules, Proc. SPIE., 2003, 4982.

20 C. Hua and E. Johnsen, Nonlinear oscillations following the rayleigh collapse of a gas bubble in a linear viscoelastic (tissue-like) medium, Physics of Fluids, 20013, 25, 083101.

21 B. Hess, C. Kutzner, D. van der Spoel and E. Lindahl, Gromacs 4: Algorithms for highly efficient, load-balanced, and scalable molecular simulation, Journal of Chemical Theory and Computation., 2008, 4, 435-447.

22 J. Hermans, H. J. C. Berendsen, W. F. Van Gunsteren and J. P. M. Postma, A consistent empirical potential for waterprotein interactions, Biopolymers., 1984, 23, 1513-1518.

23 J. P. Wahrmann, G. Drugeon, E. Delain and D. Delain, Gene expression during the differentiation of myogenic cells of the 16 line, Biochimie., 1976, 58, 551-562.

24 A. Gruzman, O. Shamni, M. Ben Yakir, D. Sandovski, A. Elgart, E. Alpert, G. Cohen, A. Hoffman, Y. Katzhendler, E. Cerasi and S. Sasson, Novel d-xylose derivatives stimulate muscle glucose uptake by activating ampactivated protein kinase alpha, J. Med. Chem., 2008, 51, 8096-8108. 